\title{
Bilateral Rotational S Flap Technique for Preventing Restenosis in Patients With Severe Circular Anal Stenosis: A Review of 2 Cases
}

\author{
Yuda Handaya ${ }^{1}$, Mukhamad Sunardi ${ }^{2}$ \\ ${ }^{1}$ Digestive Surgery Division, Department of Surgery, Faculty of Medicine, Universitas Gadjah Mada/Dr. Sardjito Hospital, Yogyakarta; ${ }^{2}$ Faculty \\ of Medicine, Universitas Gadjah Mada/Dr. Sardjito Hospital, Yogyakarta, Indonesia
}

Anal stenosis is a late hemorrhoidectomy complication. Sphincterotomy and various anoplasty techniques are used for treatment severe anal stenosis, such as the C flap, House flap, U flap, and rotational S flap, but no procedure is ideal for every patient. We review 2 cases of severe circular anal stenosis. Their complaints included narrow caliber of the stool and feeling unsatisfied defecation. Excision of scar tissue using the circular technique was followed by reconstruction using the bilateral rotational S flap procedure. At the 1-year follow-up, the patient had complaints about neither defecation nor pain, and no longer needed laxative agents. In conclusion, the bilateral rotational $\mathrm{S}$ flap technique should be considered as a viable treatment because it can also prevent the occurrence of restenosis, especially given the consideration of adequate blood supply.

Keywords: Constipation; Severe anal stenosis; Bilateral rotational S flap; Hemorrhoidectomy; Anoplasty

\section{INTRODUCTION}

Anal stenosis is one of the complications after a hemorrhoidectomy. It is an abnormal inelastic narrowing of the anal canal due to stricture of the epithelial lining, which is replaced by fibrous connective tissue [1]. The incidence of anal stenosis after a hemorrhoidectomy can be as high as 5\% [2], with $90 \%$ of the cases caused by overzealous hemorrhoidectomies [3]. Treatment, both medical and surgical, should be decided based on the severity of the stenosis $[4,5]$. In patients with severe anal stenosis, a sphincterotomy and various anoplasty techniques should be done: these techniques include scar excision, a partial internal sphincterotomy, and reshaping of the area with mucosal flaps or skin or with

Received: July 28, 2017 - Accepted: October 16, 2017

Correspondence to: Yuda Handaya, M.D.

Digestive Surgery Division, Department of Surgery, Faculty of Medicine, Universitas Gadjah Mada/Dr. Sardjito Hospital, Jl. Kesehatan No. 1

Yogyakarta 55281, Indonesia

Tel: +62274581333, Fax: +62274581333,E-mail: yudahandaya@ugm.ac.id ORCID code: https://orcid.org/0000-0003-0256-1955

(C) 2019 The Korean Society of Coloproctology

This is an open-access article distributed under the terms of the Creative Commons Attribution NonCommercial License (http://creativecommons.org/licenses/by-nc/4.0) which permits unrestricted noncommercial use, distribution, and reproduction in any medium, provided the original work is properly cited. both in various configurations [1]. Several corrective techniques for severe anal stenosis have been described in the literature [5-7]. The rotational S flap is one of those used. This manuscript reports on two cases of severe circular anal stenosis treated with this technique to prevent restenosis.

\section{CASE REPORTS}

This study has been approved by the Institutional Review Board of the Faculty of Medicine, Universitas Gadjah Mada Indonesia under permit ethic approval protocol number KE/0896/08/2018. The authors attest that full and informed consent was obtained from every single patient who have undergone medical treatment in our Hospital. The informed consent form also declared that patient data or samples will be used or educational or research purposes.

\section{Case 1}

This case involved a 47-year-old man experiencing difficulty with defecation. The patient had a history of a hemorrhoidectomy using the thermal excision (safute) technique. On the third day after the procedure, the patient complained of anal bleeding; this was improved after having undergone conservative treatment. Two 


\section{Coloporoctiogy}

weeks after surgery, the patient complained of the stool's narrow caliber and felt unsatisfied with his bowel movements. We found anal constriction on digital rectal examination when one finger could not be inserted into the anus. The patient was diagnosed as having severe anal stenosis (Fig. 1), and rotational S flap design was drawn with methylene blue (Fig. 2). We excised the scar tissue by using the circular technique (Fig. 3A); this was followed by reconstruction using the rotational $\mathrm{S}$ flap technique (Fig. 4).

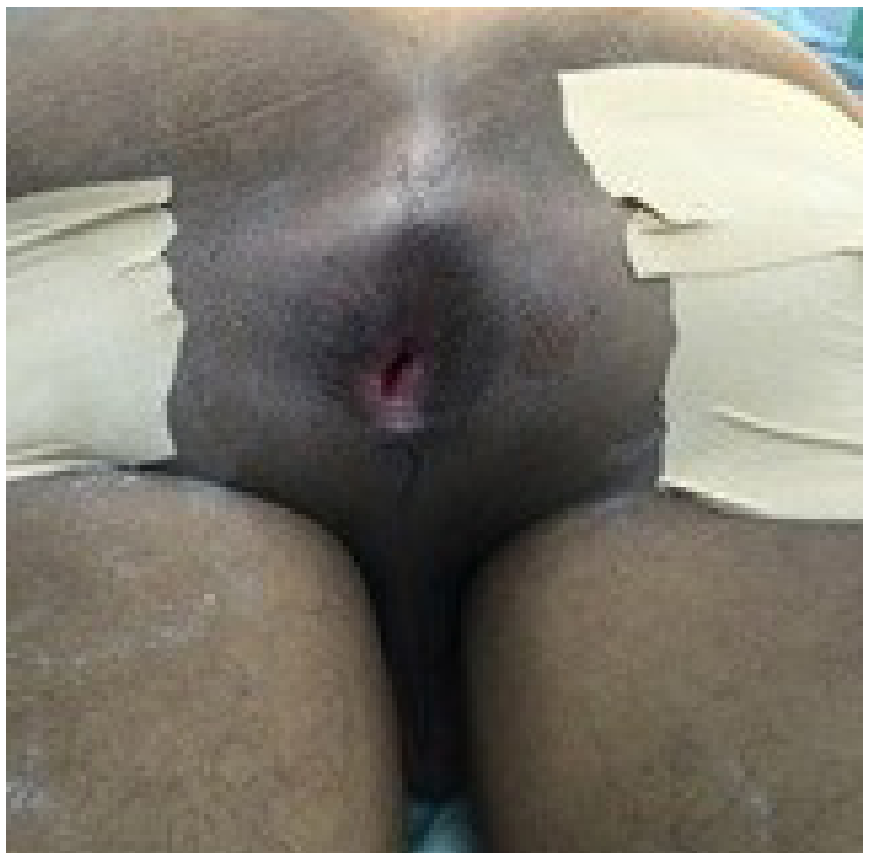

Fig. 1. Severe circular anal stenosis. Digital rectal examination showed that one finger could not be inserted into the anus.

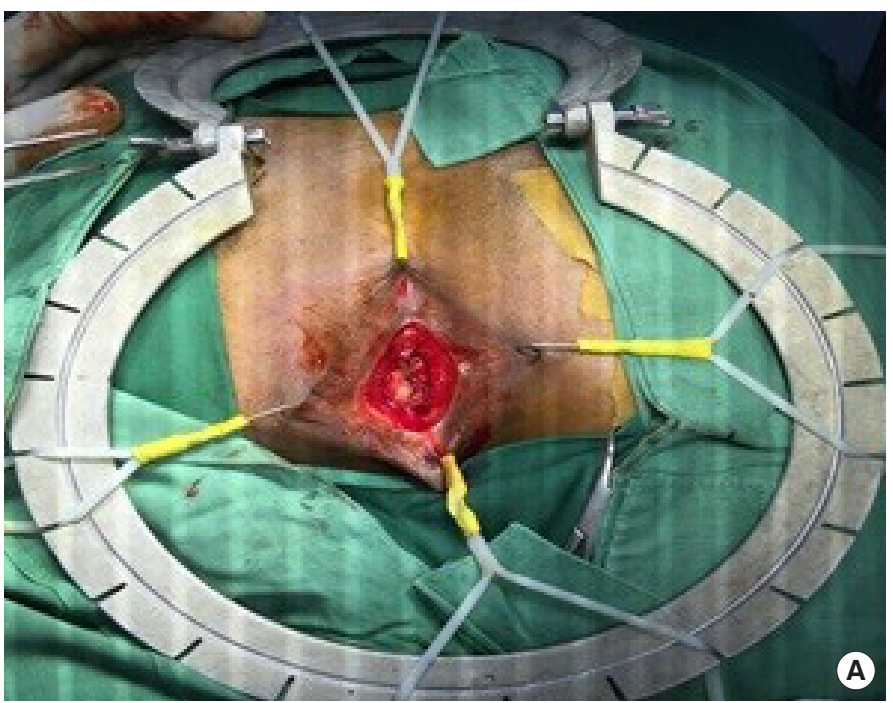

Postoperative care was adjusted to Dr. Sardjito Hospital protocol (Fig. 5). The patient was given ceftriaxone, $1 \mathrm{~g}$ intravascular injection, and metronidazole, $500 \mathrm{mg} 3$ times/day orally, for 4 days. These were followed by perianal wound care with sterile aqua and chloramphenicol ointment. The patient became an outpatient on the fifth day after surgery for perianal wound care using a potassium permanganate solution, chloramphenicol ointment, cefixime, $200 \mathrm{mg}$ twice per day, metronidazole, $500 \mathrm{mg} 3$ times per day, and paracetamol, $500 \mathrm{mg}$ orally 3 times per day.

Two weeks after surgery the patient felt a smooth consistency of the stool, but still experienced pain. At the 1-month follow-up, the patient felt smooth consistency, and pain was no longer perceived. At the 1-year follow-up, the patient had no complaints

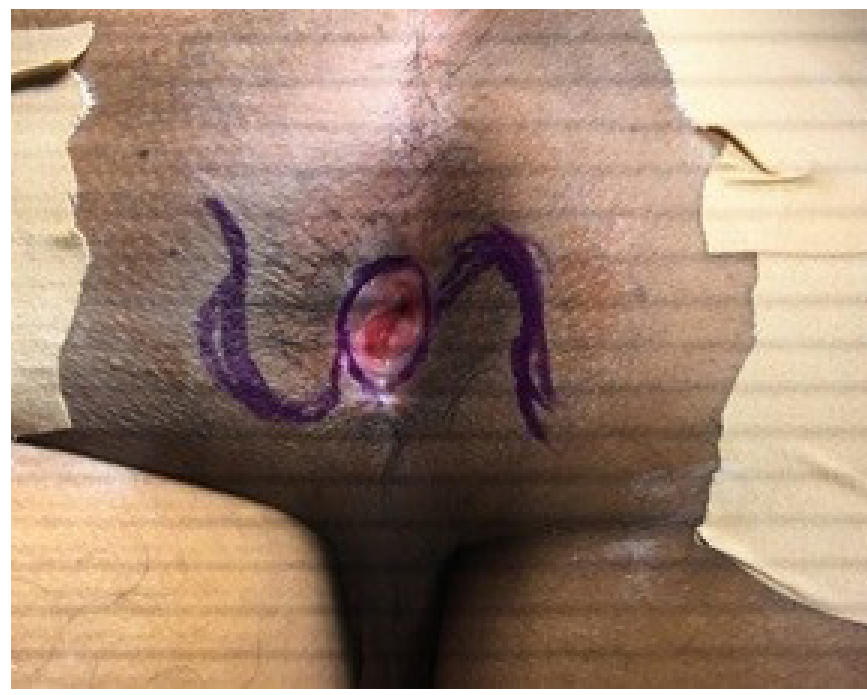

Fig. 2. Rotational S flap design was drawn with methylene blue.

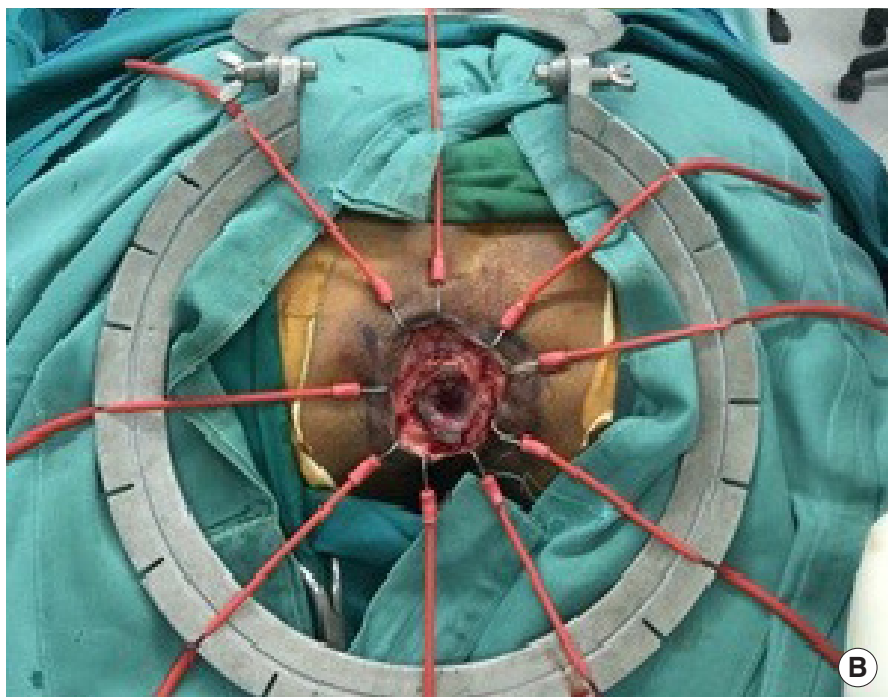

Fig. 3. Circular excision of the scar was done on 2 patients: first patient (A) and second patient (B). 


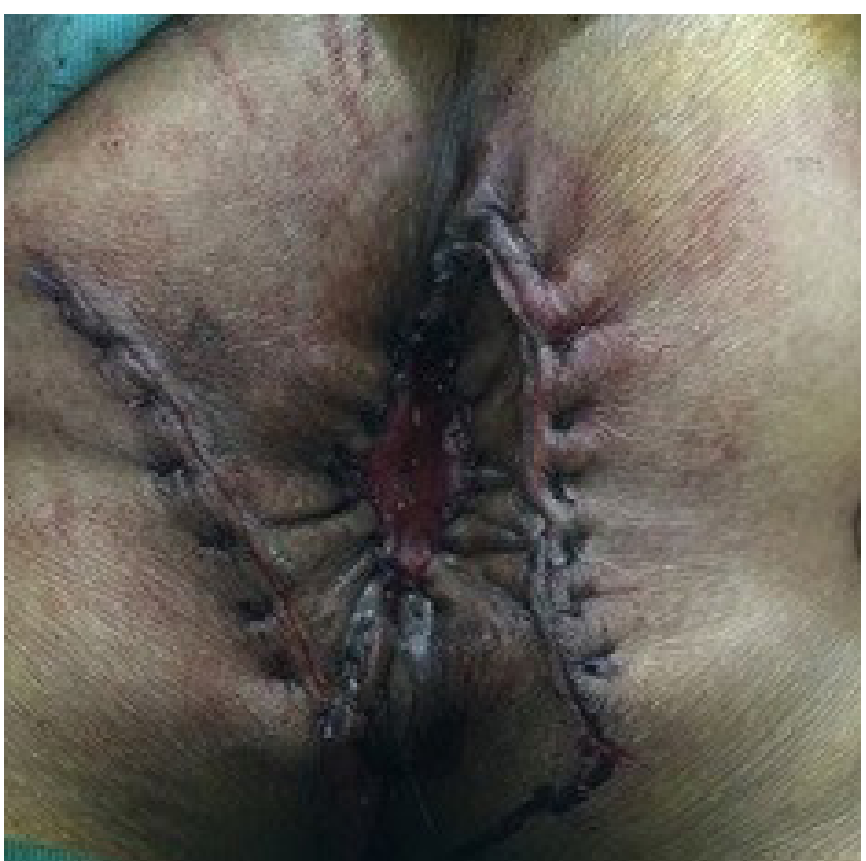

Fig. 4. Rotational S flap was performed on the patient.

about either defecation or pain, and no longer needed to use laxative agents.

\section{Case 2}

We saw a 57-year-old woman experiencing pain due to difficult defecation. She had a history of a previous hemorrhoidectomy 10 years earlier and a revision surgery 5 years earlier. After the second hemorrhoidectomy, the lump size was reduced, but she continued to feel painful defecation. The patient complained of a narrow stool with ongoing pain and unsatisfactory defecation. We found anal constriction upon digital rectal examination when one finger could not be inserted into the anus. The patient was diagnosed with severe anal stricture. We excised the scar tissue by using the circular technique (Fig. 3B); this was followed by reconstruction using the rotational $\mathrm{S}$ flap technique.

For the postoperative procedure, we used the same protocol as was used for the first case (Fig. 5). Two weeks after surgery, the patient felt smooth consistency in the stool, but pain was still being experienced. At the 1-month follow-up, the patient felt smooth consistency in the stool, and pain was no longer perceived. At the 1-year follow-up, the patient had no complaints with either defecation or pain and no longer needed laxative agents.

\section{Operative procedure}

The 2 patients were placed in the prone-jackknife position with the buttocks spread. A bilateral rotational S flap incision was performed after the double rotational S flap design had been drawn

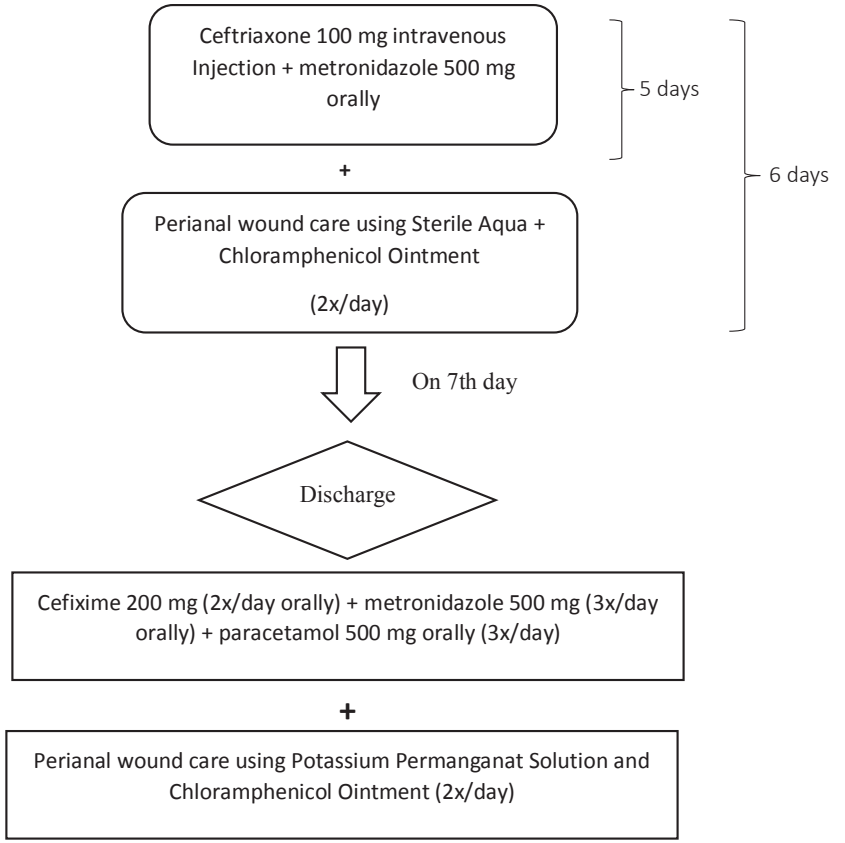

Fig. 5. Postoperative protocol for the rotational S-flap technique at Dr. Sardjito Hospital.

with methylene blue. A perianal stenosis excision was done in the fibrotic area. For the evaluation of the diameter of the anal canal, a $4-\mathrm{cm}$ busination was used. After rotation, the flap was fixed, and the subcutaneous layer was reconstructed with vicril 3.0 resorbable sutures (Johnson \& Johnson, New Brunswick, NJ, USA) while the skin was sewn with 2.0 nonresorbable sutures. During the postoperative procedures, anal spongostan was installed. The patients had an uncomplicated postoperative stay and satisfactory recovery, with no sign of morbidity.

\section{DISCUSSION}

Anal stenosis is a condition that causes patients to feel uncomfortable. Common symptoms are pain with defecation, constipation, obstipation, narrow stool caliber, and bleeding [8]. Strong evidence supports the diagnosis of anal stenosis based on symptoms, history of anal surgery, and local inspection using a digital rectal examination. In our patients, both had a history of hemorrhoidectomy procedures. These patients were diagnosed as having severe anal stenosis when the little finger could not be inserted into the anal canal upon digital rectal examination; the validity of using this criterion for diagnosing anal stenosis had been previously reported by Tahamtan et al. [9] and Milsom and Mazier [10].

For treating severe anal stenosis, we use a sphincterotomy and various anoplasty procedures. These procedures include scar excision, a partial internal sphincterotomy, and reshaping of the area with mucosal flaps, skin, or a combination of the two, in various configurations [1]. Several techniques, such as the C flap, the 
House flap, the U flap, and the rotational S flap techniques, are indicated for the treatment of severe anal stenosis [6], but one procedure is not ideal for every patient. The choice of surgery must be adjusted to the individual patient [11]. The authors selected the rotational S flap technique due to the characteristic stricture in these patients and a circumferential scar. This method can be performed bilaterally, which coincides with the work of Hudson et al. [12] and Kościński [13]; the treatment of circular anal stenosis requires a circumferential excision and the formation of 2 wide rotational flaps to cover the tissue defect.

The rotational S flap was chosen, as it has a better pedicle for preserving good vascular supply, compared to the House flap and the $\mathrm{U}$ flap techniques, as described by Brisinda et al. [6]; they maintain that the rotational S flap provides an adequate blood supply, avoids tension, and can be performed bilaterally if necessary for coverage of large areas with skin. Careful selection of anoplasty techniques according to the size and the shape of the stenosis and preservation of the vascular supply for advancement of the flap without excessive tension in the suture line can produce good outcomes, as already explained by Kościński [13].

Vascularity plays an important role in preventing hypoxic conditions. In contrast, poor perfusion creates hypoxic wounds, which disrupt the healing process. According to reports in the literature, hypoxia inducible factor-1, which is expressed in hypoxic conditions, will trigger the onset of fibrosis and scarring [14], as mentioned by Tandara and Mustoe [15]. The presence of impaired wound healing will impact the presence of fibrosis due to a buildup of fibroblasts with hypoxic features [15]. A good vascular supply can accelerate wound healing and prevent stricture occurrence. We chose the rotational S flap to promote this healing process.

Given severe circular anal stenosis, the author rated the bilateral rotational S flap as a simple technique compared to the House flap and the $\mathrm{U}$ flap approaches because both approaches must be done multiple times to reconstruct the wound after excision of the circular anal fibrosis.

In conclusion, the bilateral rotational S flap technique should be considered as a viable treatment because it can also prevent the occurrence of restenosis after treatment for circular severe anal stenosis, especially given the consideration of adequate blood supply.

\section{CONFLICT OF INTEREST}

No potential conflict of interest relevant to this article was reported.

\section{REFERENCES}

1. Casadesus D, Villasana LE, Diaz H, Chavez M, Sanchez IM, Martinez PP, et al. Treatment of anal stenosis: a 5-year review. ANZ J Surg 2007;77:557-9.

2. Blumetti J. Anal stenosis In: Abcarian H, Cintron JR, Nelson RL, editors. Complication of anorectal surgery: prevention and management. Cham (Switzerland): Springer International Publishing; 2017. p. 235-46.

3. Brisinda G. How to treat haemorrhoids. Prevention is best; haemorrhoidectomy needs skilled operators. BMJ 2000;321:582-3.

4. Liberman H, Thorson AG. How I do it. Anal stenosis. Am J Surg 2000;179:325-9.

5. Habr-Gama A, Sobrado CW, de Araújo SE, Nahas SC, Birbojm I, Nahas CS, et al. Surgical treatment of anal stenosis: assessment of 77 anoplasties. Clinics (Sao Paulo) 2005;60:17-20.

6. Brisinda G, Vanella S, Cadeddu F, Marniga G, Mazzeo P, Brandara F, et al. Surgical treatment of anal stenosis. World J Gastroenterol 2009;15:1921-8.

7. Corman ML, Bergamashi RC, Nicholls RJ, Fazio VW, editors. Corman's colon and rectal surgery. 6th ed. Philadelphia (PA): Wolters Kluwer Health/Lippincott Williams \& Wilkins; 2013. p. 327.

8. Lefta MJ, Baqir QK. Diamond flap anoplasty for severe anal stenosis. Basrah J Surg 2013;23-6.

9. Tahamtan M, Ghahramani L, Khazraei H, Tolouei YT, Bananzadeh A, Hosseini SV, et al. Surgical management of anal stenosis: anoplasty with or without sphincterotomy. J Coloproctol 2017;37: 13-7.

10. Milsom JW, Mazier WP. Classification and management of postsurgical anal stenosis. Surg Gynecol Obstet 1986;163:60-4.

11. Nugent KP. Anoplasty in operative surgery of the colon, rectum and anus. In: O'Connell PR, Madoff RD, Solomon MJ, Williams NS, editors. Operative surgery of the colon, rectum, and anus. 6th ed. Boca Raton: CRC Press; 2015. p. 195-9.

12. Hudson AT. S-plasty repair of Whitehead deformity of the anus. Dis Colon Rectum 1967;10:57-60.

13. Kościński T. Treatment of iatrogenic anal stricture. Polski Przegląd Chirurgiczny 2007;79:280-5.

14. Hong WX, Hu MS, Esquivel M, Liang GY, Rennert RC, McArdle $\mathrm{A}$, et al. The role of hypoxia-inducible factor in wound healing. Adv Wound Care (New Rochelle) 2014;3:390-9.

15. Tandara AA, Mustoe TA. Oxygen in wound healing--more than a nutrient. World J Surg 2004;28:294-300. 